Results Findings from the review indicate that the LFEs performed reviews and signed-out monthly and quarterly reports of the SRs to the PR as well as providing feedback and recommendations on these reports. The initiative has also led to immediate adjustments in financial management activities, which addresses current concerns related to accountability. The LFEs intervention has lead to improved country audit performance.

Conclusion The LFEs, under the ISEAN-Hivos Program, show strong potential to be a good practise in implementing a regional HIV grant. To enhance overall capacity building, an LFE Finance Management Manual was also developed for their reference. This manual introduces to other HIV-focused programme implementers a new concept of "embedding" LFEs among Sub-Recipients under Global Fund projects especially at a multi-country level.

\section{P6.021 CHARACTERISTICS OF HIGH RISK MSM INTO A REPEAT SELF-SAMPLING HIV-1 ANTIBODY TESTING FEASIBILITY AND ACCEPTABILITY PILOT}

doi:10.1136/sextrans-2013-051184.1175

R Elmahdi, S Fidler. Imperial College London, London, UK

Background There continues to be an increase in new HIV diagnoses amongst MSM in the UK, which contributes disproportionately to onwards transmission. In an attempt to reduce the undiagnosed fraction and encourage repeat testing amongst high risk MSM we assessed the feasibility and acceptability of enrolment into a repeat self-sampling HIV-1 antibody testing strategy between May and December 2012. In order to assess representativeness, we compared the characteristics of individuals consenting to take part in the study with those attending the clinical service.

Methods Baseline characteristics at enrolment of 50 eligible MSM attending a specialist HIV young MSM clinic were compared with a previous audit of demographics, sexual risk behaviour and HIV testing frequency of 256 clinic attendees in the year preceding study enrolment.

Results Basic demographic characteristics between the study and clinic population were comparable. There was no significant difference in the median number of reported sexual partners in the last 12 months between groups, which was $2, p=0.74$, or the proportion of those with an STI diagnosis in the last year, which was $22 \%$ amongst the study population compared to $20 \%$ in the clinic population, $p=0.74$. There was no statistically significant difference in the median number of HIV tests taken in the last year, which was 1 in both groups, $p=0.9$.

Conclusion Individuals consenting to enrol into a feasibility study of self sampling for HIV testing are comparable to the general clinic attendees and should be representative of this key risk group in later assessment of comparable frequency of HIV testing.

\section{P6.022 RESULTS OF THE GLOBAL FUND PROGRAMMES IMPLEMENTATION ON STI DIAGNOSTICS AND TREATMENT WITHIN MOST-AT-RISK POPULATIONS IN UKRAINE}

doi:10.1136/sextrans-2013-051184.1176

O Savenko, S Filippovych, Z Islam. International HIVIAIDS in Ukraine, Kiev, Ukraine

Problem: Ukraine has the fastest HIV spread pace in Europe (221 806 people as of November 2012).

Sexual HIV transmission mode has been dominant in Ukraine since 2008 (51\%-sexual, 28\%-parenteral).

HIV/STI epidemic in Ukraine is concentrated in MARPs and threatens to generalise.

Activity description:

STI diagnostics and treatment programmes for MARPs commenced in Ukraine since 2008, supported by the Global Fund to
Fight AIDS, Tuberculosis and Malaria, in an unfavourable situation due to the lack of understanding between medical services, adverse attitude to case management principles and integrated care. Several models of dermatovenerologic assistance to MARPs and stage-bystage implementation thereof were developed.

Results

In 200862 HCFs and 82 NGOs joined the programme.

In 2012 STI diagnostics and treatment are provided in 108 HCFs of Ukraine (50 skin and venereal dispensaries, 25 AIDS centres, 33 general facilities).

As of 31.012.2012 674362 screening tests and counselling for STI and viral hepatitis and 38872 STI treatment courses were provided for MARPs.

193247 MARPs representatives (as of 31.07.2012) referred to 15 mobile clinics which provide HIV/STI counselling and testing.

16 trainings were held for NGOs representatives and medical facilities.

In 201222 multidisciplinary teams were created to provide STI diagnostics and treatment services for MARPs in HCFs.

The $\mathrm{MoH}$ of Ukraine working group on amending National STI Protocols was created.

\section{Conclusions}

1. Program should be implemented simultaneously under several models considering national and regional specifics.

2. STI diagnostic and treatment programmes should become an integral part of HIV prevention services package for MARPs.

3. MDTs are the most successful model.

4. National STI Protocols amendment and case management implementation are necessary.

\section{P6.023 CROSS - BORDER HIV \& AIDS INTERVENTION PROGRAMME IN SEVEN EAST AFRICAN COUNTRIES (2008 - 2012)}

doi:10.1136/sextrans-2013-051184.1177

'A K Woldemichael, ${ }^{2} \mathrm{~F}$ Alwan, ${ }^{1} \mathrm{~A}$ Hassen, ${ }^{1} \mathrm{~F}$ Kazibwe, 'A Fadel. 'IRAPP, Kampala, Uganda, ${ }^{2} \mathrm{G} A D$, Djibouti, Djibouti

Background The IGAD Regional HIV \& AIDS Partnership Program reflects the common objective of NACs of IGAD States and partners to work in a mutually supportive way to address the subregional CBMPs aspects of the HIV/AIDS challenge. The Member countries are Djibouti, Kenya, Uganda, Ethiopia, Sudan, South Sudan and Somalia. The objective of this study was to show the progress made from 2008 until 2012.

Methodology The project was conducted in all IGAD States. The implementation at hot spots started since 2009 up to end of 2012. Joint Review Meetings conducted quarterly during the implementation phases.

Result A total of 38 sites supported by the project, of which 9 refugee camps and 29 hot spots. All sites have been enrolled following baseline assessment. Currently there are 69VCT, 63STI, 35PMTCT, and 29ART sites with community HIV/AIDS programmes. HIV tested clients reached to 292,253. A total of 1913 pregnant women found to have HIV; of these $89.6 \%$ of them have been received ARVD prophylaxis. The numbers of STI patients treated were 49133. PLHIV on chronic care reached at 15,649; while PLHIV on currently ART were 8429 and the number of patients enrolled in HBC, 408. Since the onset of the programme, a total of $2868 \mathrm{HCPs}$, 2924 peer educators, 856 youth and 6945 PLHIV, CSWs and community members have been trained. A cumulative of 6,081320 male and 98553 female condoms distributed across all IRAPP supported sites. A total of 35 PLHIV associations established in IRAPP supported sites.

Conclusion The pilot project introduced in the 7 IGAD Member States showed a good forum for continuum HIV prevention, care, treatment and support programmes for the CBMPs. The experience 